\title{
Water and contaminant flux estimation from multi-layer passive flux meter measurements
}

\author{
H. Klammler ${ }^{1,2,3}$, K. Hatfield ${ }^{2,3}$, J. Luz ${ }^{1}$, M. Annable ${ }^{3,4}$, \\ M. Newman ${ }^{2,3}$, J. Cho ${ }^{3,4}$, A. Peacock ${ }^{5}$, V. Stucker ${ }^{6}$, \\ J. Ranville ${ }^{6}$ \& C. Clark II ${ }^{7}$ \\ ${ }^{I}$ Department of Environmental Science and Sustainable Development, \\ Federal University of Bahia, Brazil \\ ${ }^{2}$ Department of Civil and Coastal Engineering, \\ University of Florida, USA \\ ${ }^{3}$ Inter-Disciplinary Program in Hydrologic Sciences, \\ University of Florida, USA \\ ${ }^{4}$ Department of Environmental Engineering Sciences, \\ University of Florida, USA \\ ${ }^{5}$ Microbial Insights, Inc., Rockford, USA \\ ${ }^{6}$ Department of Chemistry and Geochemistry, \\ Colorado School of Mines, USA \\ ${ }^{7}$ Department of Civil and Environmental Engineering, \\ Florida A\&M University, USA
}

\begin{abstract}
The passive flux meter (PFM) enables the measurement of cumulative water and contaminant mass fluxes in porous aquifers. It consists of a sorbent material, which is installed in a monitoring well to intercept groundwater flow. Tracer losses and contaminant retention on the sorbent are used to estimate water and contaminant mass fluxes through the device. In the multi-layer PFM different (sorbent) materials are used in an annulus (layer-type) configuration. This allows leached tracers inside the PFM (no tracer release into aquifer) to be retained and facilitates simultaneous deployment of different sorbent types in a single device. In order to estimate undisturbed ambient fluxes in the aquifer, measurements need to be corrected for flow convergence or divergence induced by the well and PFM components. We make use of an analytical solution to the potential flow
\end{abstract}


problem of uniform flow disturbed by a system of concentric rings of contrasting hydraulic conductivities. A flow convergence factor is defined as a function of PFM ring conductivities and radii, where tracer elution and contaminant sorption may occur in arbitrary layers. The results are used for calibration of convergence factors of a multi-layer PFM to laboratory sand box experiments.

Keywords: aquifer, groundwater, plume, flow convergence, sand box.

\section{Introduction}

Groundwater contamination is recognized as a dangerous threat to ecosystems and human drinking water supplies. Besides contaminant concentrations (mass per volume), contaminant mass fluxes (mass per cross sectional area per time) have been used more recently as relevant measures for contaminant source identification, risk assessment, decision making and remediation performance control (ITRC [1]). Currently, three fundamental approaches are available for measuring contaminant fluxes: (1) Multi-level sampling (MLS; Einarson and Mackay [2]), which is based on separate measurements of contaminant concentrations and water fluxes for subsequent multiplication to obtain contaminant fluxes. (2) Integral pump tests (IPT; Bockelmann et al. [3]), which extract contaminated groundwater from the aquifer through pumping from a well and monitor contaminant concentrations at the well head over time. (3) Passive flux meter measurements (PFM; Hatfield et al. [4], Annable et al. [5]) based on the installation of sorbent materials in observation wells, where the sorbents initially contain known amounts of resident tracers. From detected tracer losses from a sorbent and contaminant masses sorbed onto a sorbent through laboratory analyses, cumulative (i.e., time integrated or averaged over the period of installation) water and contaminant fluxes may be obtained simultaneously as depth profiles along the well.

PFMs have typically been deployed in observation wells as self-contained units consisting of a single sorbent material, which acts as both a leaching tracer reservoir and a contaminant trap. As a consequence, it has to be assured that (1) the sorption properties of the sorbent material are appropriate for both tracer(s) and target contaminants, and (2) that the chemical properties of the tracer(s) are such that tracer release (even though minimal) into the aquifer does not cause legal or environmental problems. In an effort to circumvent these two issues (e.g., for measuring water and contaminant fluxes at Rifle, CO, USA) a multilayer PFM has been developed and tested, which consists of multiple concentric rings (layers) of different materials (which may be sorbents or not).

Figure 1 compares the two different PFM configurations, where figure 1a shows a single sorbent installed inside a well screen, while figure $1 \mathrm{~b}$ illustrates the annular composition of a multi-layer PFM installed in a screened well. From the periphery towards the center $k_{i}[\mathrm{~L} / \mathrm{T}]$ and $r_{i}[\mathrm{~L}]$ denote the hydraulic conductivities and outer radii of the different rings. $k_{0}$ corresponds to the aquifer, $k_{1}$ is the well screen, $k_{2}$ an outer sorbent layer for contaminant sorption (e.g., Lewatit resin for uranium), $k_{3}$ an intermediate sorbent layer (e.g., granular activated carbon (GAC) for alcoholic tracers) to retain tracers eluded from an 
inner sorbent layer of $k_{5}$ (e.g., also consisting of GAC for alcoholic tracers). Between the two GAC layers, there is a thin perforated stainless steel pipe of conductivity $k_{4}$, which serves to separate the inner GAC from the outer one for installation and laboratory analysis. The center circle of radius $r_{6}$ is an impermeable pipe for physical stabilization and water evacuation during PFM
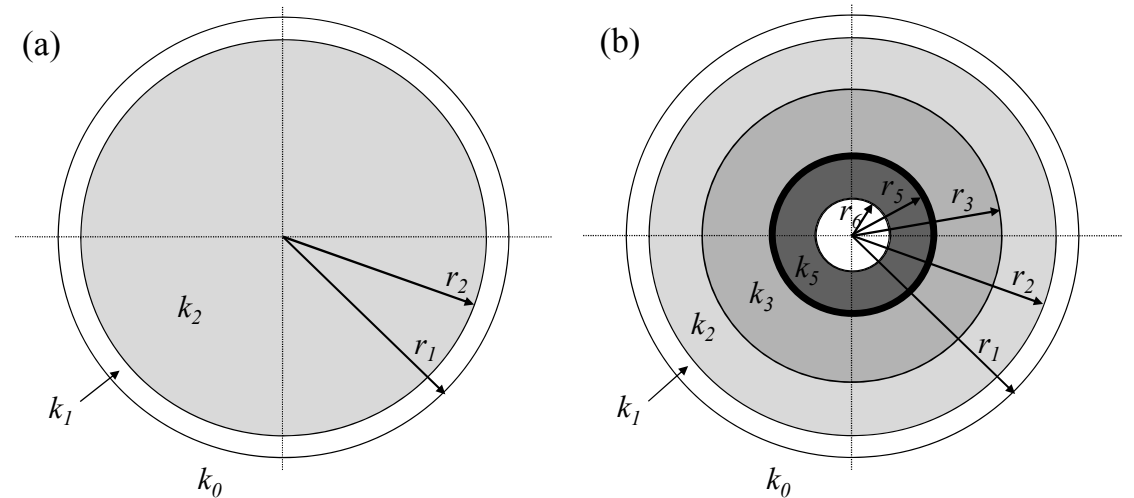

Figure 1: Horizontal cross sections of well screens and (a) a PFM consisting of a single sorbent and (b) a multi-layer PFM as used in laboratory testing. Bold black circle is of outer radius $r_{4}$ and conductivity $k_{4}$.

Table 1: $\quad$ Summary of multi-layer PFM properties as depicted in figure $1 \mathrm{~b}$ with $k_{1}$ and $k_{4}$ to be determined from laboratory box experiments.

\begin{tabular}{|c|c|c|c|}
\hline $\begin{array}{c}\text { Ring } \mathrm{n}^{0} . i \\
{[-]}\end{array}$ & $\begin{array}{c}\text { Conductivity } k_{i} \\
{[\mathrm{~m} / \text { day }]}\end{array}$ & $\begin{array}{c}\text { Outer radius } r_{i} \\
{[\mathrm{~cm}]}\end{array}$ & Material / purpose \\
\hline 0 & 33 & infinite & Aquifer (sand) \\
\hline 1 & $k_{1}$ & 5.7 & Well screen (slotted PVC pipe) \\
\hline 2 & 250 & 5.1 & Lewatit resin for uranium sorption \\
\hline 3 & 350 & 3.8 & GAC for tracer retention \\
\hline 4 & $k_{4}$ & 2 & Perforated pipe for separation \\
\hline 5 & 350 & 1.9 & GAC for tracer elution \\
\hline 6 & 0 & 0.8 & Impermeable center pipe \\
\hline
\end{tabular}

installation and removal. Table 1 summarizes the properties of the multi-layer PFM configuration used in laboratory sand box experiments for determination of $k_{l}$ and $k_{4}$ and subsequent deployment for measuring water and uranium fluxes at the uranium field site in Rifle. 
Similar to the simple PFM, the multi-layer PFM provides data in terms of tracer losses from sorbent ring 5 and contaminant masses retained from sorbent ring 2. Following the method of Hatfield et al. [4] the apparent flux $q_{P F M, i}[\mathrm{~L} / \mathrm{T}]$ through the $i$-th ring of a PFM may be found as

$$
q_{P F M, i}=\frac{\left(1-m_{r i}\right)\left(r_{i}^{2}-r_{i+1}^{2}\right) \pi \theta_{i} R_{i}}{2 r_{i} t}
$$

where $m_{r i}[-]$ is the relative mass of a tracer remaining (with respect to the initial mass of that tracer) after time of exposure $t[\mathrm{~T}]$ in the $i$-th ring of relative water content $\theta_{i}[-]$ and retardation factor $R_{i}[-]$. The attribute "apparent" indicates that flow is generally not uniform inside a multi-layer PFM and $q_{P F M, i}$ is to be understood as a discharge per unit transect area of the $i$-th ring perpendicular to incident flow direction. In analogy, an apparent contaminant mass flux $J_{P F M, i}$ $\left[\mathrm{M} /\left(\mathrm{TL}^{2}\right)\right]$ trough the $i$-th ring may be obtained from

$$
J_{P F M, i}=\frac{M_{s i}}{2 r_{i} b t}
$$

where $M_{s i}[\mathrm{M}]$ is the mass of contaminant sorbed in the $i$-th layer over a PFM interval of length $b[\mathrm{~L}]$. Eqn (1) is valid as long as none of the stream tubes through the $i$-th layer are completely cleared (empty) of tracer, while eqn (2) is valid as long as none of the contaminant previously sorbed onto the $i$-th layer is again released from it. As a consequence, within their ranges of validity eqns (1) and (2) do not depend on the properties (e.g., non-uniformity) of the flow field in the respective rings. Note that for $r_{i+1}=0$ (i.e., the $i$-th layer is the center circle) eqn (1) reduces to eqn (18) of Hatfield et al. [4], whose coefficient of 1.67 appears as $\pi / 2 \approx 1.57$ here. This is a consequence of the range of validity stipulated (an analogous observation applies to eqn (2)).

However, due to the more complex configuration of the multi-layer PFM and flow refraction between layers of different conductivities, an assessment of undisturbed ambient water and contaminant fluxes in the aquifer is not straightforward. Klammler et al. [6] present an analytical solution to the potential flow problem through the multi-layer PFM and they develop flow convergence factors for estimation of undisturbed (uniform) ambient fluxes. These factors, however, are with respect to the inner-most ring (center circle) only and are not applicable to other rings. The present work generalizes the convergence factors of Klammler et al. [6] to arbitrary layers in a multi-layered PFM configuration and uses the result for calibration of unknown parameters (well screen and perforated pipe conductivities $k_{1}$ and $k_{4}$ ) through a laboratory sand box experiment, such that they may be applied to the multi-layer PFM deployment at Rifle for measuring water and uranium fluxes.

\section{General flow field solution}

The solution of Klammler et al. [6] is based on potential flow through porous media (Strack [7]). It makes use of a flow field analogy between uniform flow 
disturbed by a cylindrical inhomogeneity in hydraulic conductivity and uniform flow disturbed by an impermeable or infinitely permeable cylinder. Its application is best illustrated by a numerical example, for which we use the flow domain of figure $1 \mathrm{~b}$ (table 1 ) with values of $k_{1}=2.3 \mathrm{~m} / \mathrm{d}$ and $k_{4}=3.2 \mathrm{~m} / \mathrm{d}$. This leads to the flow field (stream lines) of figure 2 with the stream function $\Psi_{i}$ $\left[\mathrm{L}^{3} / \mathrm{T}\right]$ in the $i$-th ring given by

$$
\Psi_{i}=q_{i} r b\left(1-\frac{a_{i}^{2}}{r^{2}}\right) \sin \gamma
$$

where $r[\mathrm{~L}]$ and $\gamma[-]$ are the radial and angular coordinates, respectively, $b[\mathrm{~L}]$ is the thickness of the flow domain (length of PFM interval in eqn (2)) and the parameters $a_{i}[\mathrm{~L}]$ and $q_{i}[\mathrm{~L} / \mathrm{T}]$ are given in table 2 and obtained as follows: Starting with an initial value of $a_{6}=0$ in table 2 , the columns of $k_{i a}[\mathrm{~L} / \mathrm{T}]$ and $a_{i}$ are populated from bottom up by alternately applying eqns (4) and (5). Subsequently, the column of $q_{i}$ is populated from top down by consecutive use of eqn (6). Most parameters in table 2 are auxiliary variables without direct physical equivalences (some of them complex/ imaginary). Exceptions are $q_{0}$ (undisturbed flux in the aquifer; assumed uniform), $k_{6 a}$ (equal to conductivity $k_{6}$ of inner ring), $k_{0 a}$ (equal to aquifer conductivity $k_{0}$ ) and $q_{6}$ (specific discharge in inner ring). Since $k_{6}=0$, it is further seen that $a_{5}$ is equal to the center pipe radius $r_{6}$. Following this example, the step-wise solution scheme of eqns (3) through (6) is generally applicable to an arbitrary number of layers.

$$
\begin{gathered}
k_{i a}=k_{i} \frac{1-\left(\frac{a_{i}}{r_{i}}\right)^{2}}{1+\left(\frac{a_{i}}{r_{i}}\right)^{2}} \\
a_{i-1}=r_{i} \sqrt{\frac{1-\frac{k_{i a}}{k_{i-1}}}{1+\frac{k_{i a}}{k_{i-1}}}} \\
q_{i}=q_{i-1} \frac{k_{i a}}{1+\frac{k_{i-1}}{k_{i a}}}
\end{gathered}
$$




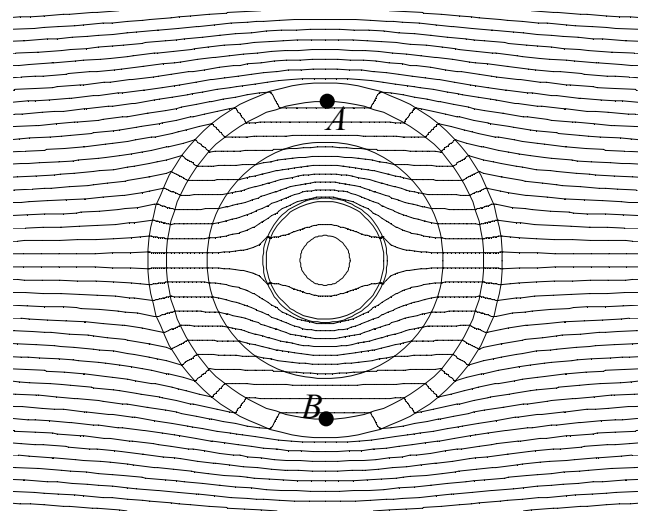

Figure 2: $\quad$ Flow field solution depicted as stream lines (lines of constant $\Psi_{i}$ ) for flow domain of figure $1 \mathrm{~b}$ (table 1$)$.

Table 2: $\quad$ Summary of flow field parameters and convergence factors for multi-layer PFM of figure $1 \mathrm{~b}$ for $k_{1}=2.3 \mathrm{~m} / \mathrm{d}$ and $k_{4}=3.2 \mathrm{~m} / \mathrm{d}$. $j=\sqrt{-1}$ is the imaginary unit.

\begin{tabular}{|c|c|c|c|c|}
\hline Ring $^{\text {o }} . i[-]$ & $a_{i}[\mathrm{~cm}]$ & $k_{i a}[\mathrm{~m} /$ day $]$ & $q_{i} / q_{0}[-]$ & $\alpha_{i}[-]$ \\
\hline 0 & 2.94 & 33 & $\boldsymbol{q}_{0} / \boldsymbol{q}_{0}=\mathbf{1}$ & 1 \\
\hline 1 & $5.05 \mathrm{j}$ & 19.12 & 0.41 & 0.73 \\
\hline 2 & 0.79 & 238.38 & 0.83 & 0.81 \\
\hline 3 & 1.73 & 229.46 & 1.01 & 0.80 \\
\hline 4 & $1.88 \mathrm{j}$ & 49.78 & 0.13 & 0.25 \\
\hline 5 & 0.80 & 244.59 & 0.32 & 0.26 \\
\hline 6 & $\boldsymbol{a}_{\mathbf{6}}=\mathbf{0}$ & 0 & 0 & 0 \\
\hline
\end{tabular}

\section{Flow convergence factors for arbitrary layers}

Using the solution of $\Psi_{i}$ from eqn (3) for an arbitrary number of layers, the apparent flux $q_{P F M, i}[\mathrm{~L} / \mathrm{T}]$ through the $i$-th ring of eqn (1) may be found. It is equal to the difference of the stream function between the lateral-most points (e.g., for $i=2$ points $A$ and $B$ in figure 2) of the ring divided by the cross sectional area perpendicular to flow.

$$
q_{P F M, i}=\frac{\Psi_{i}\left(r_{i}, \frac{\pi}{2}\right)-\Psi_{i}\left(r_{i},-\frac{\pi}{2}\right)}{2 r_{i} b}=\frac{\Psi_{i}\left(r_{i}, \frac{\pi}{2}\right)}{r_{i} b}=q_{i}\left(1-\frac{a_{i}^{2}}{r_{i}^{2}}\right)
$$


In the undisturbed aquifer the flow is assumed to be of uniform flux $q_{0}$, such that a flow convergence (or divergence) factor $\alpha_{i}[-]$ for the $i$-th ring may be defined by

$$
\alpha_{i}=\frac{q_{P F M, i}}{q_{0}}=\frac{q_{i}}{q_{0}}\left(1-\frac{a_{i}^{2}}{r_{i}^{2}}\right)
$$

In other words, the flow convergence factor expresses how much flow crosses the $i$-th ring of a multi-layer PFM with respect to the ambient flow through a ring of the same size, if the aquifer was not disturbed by the well and PFM components. A value of $\alpha_{i}$ larger than one may be viewed as a situation of flow convergence, while a value smaller than one reflects a condition of flow divergence. For a single layer PFM, flow convergence occurs when the PFM sorbent is more permeable than the aquifer and flow divergence occurs when the contrary is true. For multi-layer PFMs, however, $\alpha_{i} \geq 1$ and $\alpha_{i} \leq 1$ may occur simultaneously in different layers of a single device depending on the sequence of $k_{i}$. Returning to the example of figure $1 \mathrm{~b}$ and using values of $r_{i}$ from table 1 with values of $q_{i} / q_{0}$ and $a_{i}$ from table 2 , the values of $\alpha_{i}$ as given in the last column of table 2 are directly obtained from eqn (8) (note hereby that some values of $a_{i}$ are imaginary).

The flow convergence factor $\alpha_{i}$ from eqn (8) is a generalization over that of Klammler et al. [6], because it is applicable to any layer. If $i$ is equal to the total number of layers present, such that $\alpha_{i}$ applies to the center circle of the flow domain as shown in figure 1 , for example, then eqn (8) becomes equal to $\alpha_{i}$ from Klammler et al. [6]. Moreover, for $i=1,2$ and 3 (and an arbitrary total number of layers), eqns (3), (12) and (13) of Klammler et al. [6] may be written in a generalized form by using $k_{l a}, k_{2 a}$ and $k_{3 a}$ from eqn (4) instead of $k_{1}, k_{2}$, and $k_{3}$ resulting in

$$
\begin{gathered}
\alpha_{1}=\frac{2}{1+\frac{k_{0}}{k_{1 a}}} \\
\alpha_{2}=\frac{4}{\left(1+\frac{k_{0}}{k_{1}}\right)\left(1+\frac{k_{1}}{k_{2 a}}\right)+\left(1-\frac{k_{0}}{k_{1}}\right)\left(1-\frac{k_{1}}{k_{2 a}}\right)\left(\frac{r_{2}}{r_{1}}\right)^{2}} \\
\alpha_{3}=\frac{8}{\left(1+\frac{k_{0}}{k_{1}}\right)\left(1+\frac{k_{1}}{k_{2}}\right)\left(1+\frac{k_{2}}{k_{3 a}}\right)+\left(1-\frac{k_{0}}{k_{1}}\right)\left(1-\frac{k_{1}}{k_{2}}\right)\left(1+\frac{k_{2}}{k_{3 a}}\right)\left(\frac{r_{2}}{r_{1}}\right)^{2}+\left(1+\frac{k_{0}}{k_{1}}\right)\left(1-\frac{k_{1}}{k_{2}}\right)\left(1-\frac{k_{2}}{k_{3 a}}\right)\left(\frac{r_{3}}{r_{2}}\right)^{2}+\left(1-\frac{k_{0}}{k_{1}}\right)\left(1+\frac{k_{1}}{k_{2}}\right)\left(1-\frac{k_{2}}{k_{3 a}}\right)\left(\frac{r_{3}}{r_{1}}\right)^{2}}
\end{gathered}
$$

For $i=4$, the result of Appendix B in Klammler et al. [6] is incomplete and after substituting $k_{4}$ by $k_{4 a}$ it should be

$$
\alpha_{4}=\frac{16}{A+B+C+D+E+F+G+H}
$$


with

$$
\begin{aligned}
& A=\left(1+\frac{k_{0}}{k_{1}}\right) \cdot\left(1+\frac{k_{1}}{k_{2}}\right) \cdot\left(1+\frac{k_{2}}{k_{3}}\right) \cdot\left(1+\frac{k_{3}}{k_{4 a}}\right) \\
& B=\left(1-\frac{k_{0}}{k_{1}}\right) \cdot\left(1-\frac{k_{1}}{k_{2}}\right) \cdot\left(1+\frac{k_{2}}{k_{3}}\right) \cdot\left(1+\frac{k_{3}}{k_{4 a}}\right) \cdot\left(\frac{r_{2}}{r_{1}}\right)^{2} \\
& C=\left(1+\frac{k_{0}}{k_{1}}\right) \cdot\left(1-\frac{k_{1}}{k_{2}}\right) \cdot\left(1-\frac{k_{2}}{k_{3}}\right) \cdot\left(1+\frac{k_{3}}{k_{4 a}}\right) \cdot\left(\frac{r_{3}}{r_{2}}\right)^{2} \\
& D=\left(1-\frac{k_{0}}{k_{1}}\right) \cdot\left(1+\frac{k_{1}}{k_{2}}\right) \cdot\left(1-\frac{k_{2}}{k_{3}}\right) \cdot\left(1+\frac{k_{3}}{k_{4 a}}\right) \cdot\left(\frac{r_{3}}{r_{1}}\right)^{2} \\
& E=\left(1+\frac{k_{0}}{k_{1}}\right) \cdot\left(1+\frac{k_{1}}{k_{2}}\right) \cdot\left(1-\frac{k_{2}}{k_{3}}\right) \cdot\left(1-\frac{k_{3}}{k_{4 a}}\right) \cdot\left(\frac{r_{4}}{r_{3}}\right)^{2} \\
& F=\left(1+\frac{k_{0}}{k_{1}}\right) \cdot\left(1-\frac{k_{1}}{k_{2}}\right) \cdot\left(1+\frac{k_{2}}{k_{3}}\right) \cdot\left(1-\frac{k_{3}}{k_{4 a}}\right) \cdot\left(\frac{r_{4}}{r_{2}}\right)^{2} \\
& G=\left(1-\frac{k_{0}}{k_{1}}\right) \cdot\left(1+\frac{k_{1}}{k_{2}}\right) \cdot\left(1+\frac{k_{2}}{k_{3}}\right) \cdot\left(1-\frac{k_{3}}{k_{4 a}}\right) \cdot\left(\frac{r_{4}}{r_{1}}\right)^{2} \\
& H=\left(1-\frac{k_{0}}{k_{1}}\right) \cdot\left(1-\frac{k_{1}}{k_{2}}\right) \cdot\left(1-\frac{k_{2}}{k_{3}}\right) \cdot\left(1-\frac{k_{3}}{k_{4 a}}\right) \cdot\left(\frac{r_{2} r_{4}}{r_{1} r_{3}}\right)^{2}
\end{aligned}
$$

Note that eqns (9) through (13) are equivalent formulations to the step-wise procedure of eqns (4) through (6). While closed form expressions of $\alpha_{i}$ for $i>4$ may be derived, they become increasingly lengthy and computational implementation of eqns (4) through (6) (e.g., in a spreadsheet) may be more convenient.

Not immediately obvious from eqns (9) through (13) and more easily verifiable using eqns (5), (6) and (8) is that the ratio $\alpha_{i} / \alpha_{i-1}$ is only a function of $k_{m}$ and $r_{m}$, where $m \geq i-1$. More intuitively, this means that flow refraction between two adjacent layers does not depend on the radii and conductivities of any outside layers or the aquifer. This may be convenient for comparing flux estimates from different layers independent of the perhaps uncertain aquifer conductivity $k_{0}$ and will be explored for estimating $k_{1}$ and $k_{4}$ below. However, it also precludes the possibility of estimating an unknown $k_{0}$ from two flux estimates in different layers (as may be attempted in analogy to the method presented in Klammler et al. [6]).

Assuming that contaminant transport is dominated by advection (i.e., contaminant particles travel along the same stream tubes as water particles and effects of diffusion and dispersion are neglected), $q_{P F M, i}$ and $q_{0}$ in the first equality of eqn (8) may be substituted by $J_{P F M, i}$ from eqn (2) and the undisturbed ambient contaminant mass flux $J_{0}\left[\mathrm{M} /\left(\mathrm{L}^{2} \mathrm{~T}\right)\right]$, respectively. It is recalled that $m_{r i}$ for estimation of $q_{P F M, i}$ and $M_{s i}$ for estimation of $J_{P F M, i}$ do not have to stem from the same PFM layer (i.e., index $i$ in eqns (1) and (2) generally takes different 
values). However, if $m_{r i}$ and $M_{s i}$ are obtained from the same layer, then the same flow convergence factor $\alpha_{i}$ applies.

\section{Laboratory experiments and calibration}

For application of the multi-layer PFM configuration of figure $1 \mathrm{~b}$ at the uranium site in Rifle and determination of unknown conductivities $k_{l}$ and $k_{4}$, laboratory sand box experiments are performed. Box size is $39 \times 30.5 \times 17.9 \mathrm{~cm}$ (length $L \mathrm{x}$ width $W \times$ height $H$ ) and table 1 contains the sand conductivity as well as well screen and PFM parameters. "Ambient" water fluxes $q_{0}$ and uranium fluxes $J_{0}$ through the box are obtained from

$$
\begin{aligned}
& q_{0}=\frac{Q_{0}}{W H} \\
& J_{0}=q_{0} C_{u}
\end{aligned}
$$

where $Q_{0}\left[\mathrm{~L}^{3} / \mathrm{T}\right]$ is the independently measured water discharge through the box and $C_{u}\left[\mathrm{M} / \mathrm{L}^{3}\right]$ is the uranium concentration in the influent water. Eleven tests of different durations were run for water flux obtaining estimates $q_{P F M, 5}$ from eqn (1) for comparison to $q_{0}$ of eqn (14). In five of these tests uranium was added at $C_{u} \approx 200 \mu \mathrm{g} / 1$ for comparison of $J_{P F M, 2}$ from eqn (2) with $J_{0}$ of eqn (15).

Table 3: $\quad$ Summary of results from sand box experiments.

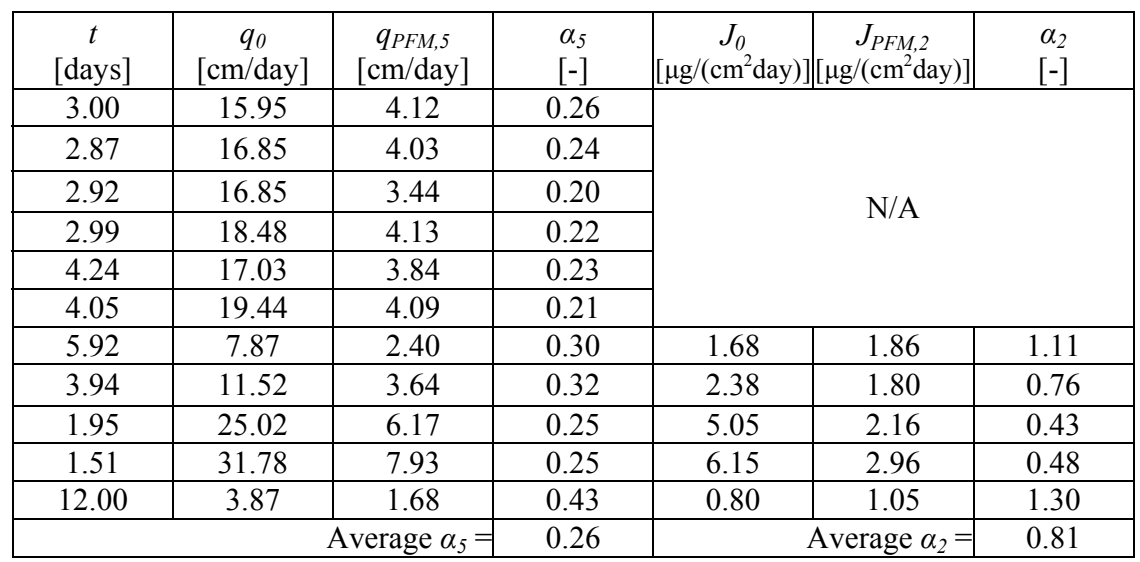

Table 3 summarizes the results of the sand box experiments and indicates average values of $\alpha_{2}=J_{P F M, 2} / J_{0}=0.81$ and $\alpha_{5}=q_{P F M, 5} / q_{0}=0.26$. At this point we return to table 2 and revert the previous assumption that the conductivities $k_{l}$ and $k_{4}$ of the well screen and the perforated pipe are known. Instead, the experimental values of $\alpha_{2}$ and $\alpha_{5}$ are used to determine effective values of $k_{1}$ and $k_{4}$ to be used in the interpretation of field deployments. For this purpose, 
advantage is taken of the previous conclusion that the ratio $\alpha_{5} / \alpha_{2}=0.32$ is not affected by $k_{l}$. Thus, $k_{4}$ may be directly found, which is most conveniently achieved by systematically varying $k_{4}$ for an arbitrary value of $k_{l}$ and observing the results in terms of $\alpha_{5} / \alpha_{2}$. Figure 3 shows the outcome of this process and leads to two possible values of $k_{4}=3.2$ and $18851 \mathrm{~m} /$ day. For each of the values found for $k_{4}$, the same process is repeated with $k_{1}$ to reach the required value of $\alpha_{2}=0.81$ (or equally $\alpha_{5}=0.26$ ). This is illustrated in figure 4 and shows that again two values of $k_{1}$ may be combined with each value of $k_{4}$, thus resulting in the four solutions given in the first two rows of table 4 .

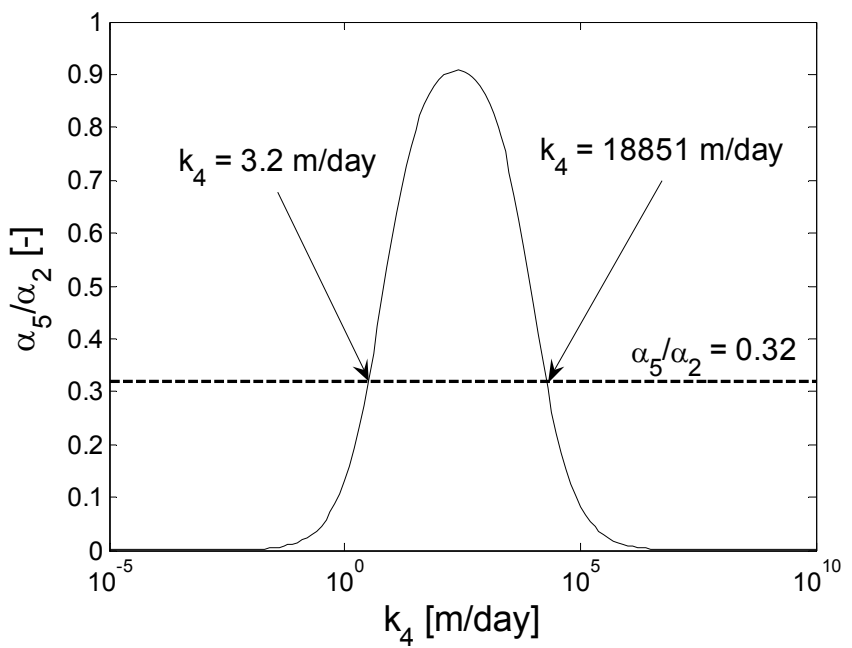

Figure 3: $\quad \alpha_{5} / \alpha_{2}$ as a function of $k_{4}$ for arbitrary $k_{1}$ to achieve target value $\alpha_{5} / \alpha_{2}=0.32$.
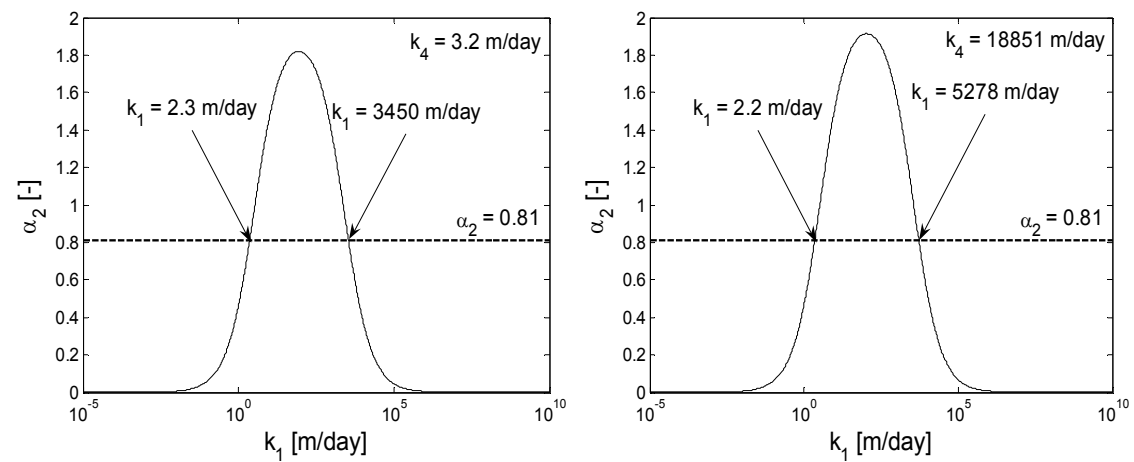

Figure 4: $\quad \alpha_{2}$ as a function of $k_{1}$ for $k_{4}=3.2$ and $18851 \mathrm{~m} /$ day to achieve target value $\alpha_{2}=0.81$. 
Table 4: Possible combinations of $k_{1}$ and $k_{4}$ in $\mathrm{m} /$ day to achieve $\alpha_{2}=0.81$ and $\alpha_{5}=0.26$ from box experiments and resulting $\alpha_{q}$ and $\alpha_{J}$ for the Rifle deployment.

\begin{tabular}{|l|l|l|l|l|}
\hline$k_{1}$ & 2.3 & 3450 & 2.2 & 5278 \\
\hline$k_{4}$ & \multicolumn{2}{|c|}{3.2} & \multicolumn{2}{c|}{18851} \\
\hline$\alpha_{q}$ & 0.20 & 0.36 & 0.20 & 0.37 \\
\hline$\alpha_{J}$ & 0.61 & 1.13 & 0.61 & 1.16 \\
\hline
\end{tabular}

For each of the four solutions pairs, a flow convergence factor $\alpha_{q}$ for water flux and a flow convergence factor $\alpha_{J}$ for uranium may be computed for the deployment conditions at the Rifle site. These conditions are identical to those of figure $1 \mathrm{~b}$, except for the presence of a filter pack of radius $10.2 \mathrm{~cm}$ and conductivity $160 \mathrm{~m} /$ day around the well screen and inside an aquifer of conductivity of approximately $2.5 \mathrm{~m} / \mathrm{d}$. Considering these modifications, results for $\alpha_{q}$ and $\alpha_{J}$ from application of eqns 4, 5, 6 and 8 are given in the last two rows of table 4 . It may be seen that $\alpha_{q} / \alpha_{J}=\alpha_{5} / \alpha_{2}=0.32$ remains constant and, more interestingly, that the solutions are pair wise identical (up to chart reading and rounding errors). It appears that this is not a coincidence as the same behaviour may be observed for other hypothetical values of aquifer and filter pack conductivities and radii. From the remaining two solution, $\alpha_{q}=0.20$ and $\alpha_{J}=$ 0.61 are proposed for use at the Rifle site, since they are associated with a low value of $k_{1}=2.3$ (and either value of $k_{4}$ ). This choice is justified by two related arguments: (1) From an independent borehole dilution test (no PFM installed) in the sand box a value of $k_{l}=87 \mathrm{~m} /$ day is estimated. This may be regarded as an upper bound for $k_{1}$ as flow in the vicinity of an open borehole is radial. Flow components in the tangential direction through the well screen are hindered by the fact that screen slots are not continuous along the circumference of a PVC screen. (2) As illustrated by the flow field in figure 2 , low $k_{l}$ and $k_{4}$ cause flow in the respective rings to be essentially radial, which is in agreement with the geometric properties of the screen slots and the pipe perforations acting as water conduits. Large values of $k_{1}$ and $k_{4}$, in turn, would lead to a certain degree of flow short circuiting along these rings, which is considered less plausible, particularly since the sorbents are granular and tend to settle into the well and close possible voids along the pipe or screen surfaces.

\section{Summary}

Based on an existing solution to the potential flow problem of uniform flow disturbed by an arbitrary number of concentric rings of contrasting conductivities, flow convergence (or divergence) factors are developed for interpretation of multi-layer PFM measurements. Flow convergence factors convert water and contaminant fluxes measured in an arbitrary layer of the PFM into estimates of respective undisturbed ambient fluxes (i.e., unaffected by the 
presence of well and PFM). Using the results in combination with laboratory sand box experiments, effective conductivities of a well screen and another separation screen between layers are determined. With these conductivities, flow convergence factors for measuring water and uranium fluxes at a site in Rifle, CO, USA, are proposed.

\section{Acknowledgements}

This research was partially funded by the first author's fellowship of the Bahia State Science Foundation (FAPESB; DCR 0001/2009), Brazil, the U.S. National Science Program (award number 0804134), the Environmental Remediation Science Program (ERSP), U.S. Department of Energy (grant number DE-FG0208ER64585), the U.S. Department of Defense (project number ER0831) under the Environmental Security Technology Compliance Program (ESTCP) and the Florida Water Resources Research Center under a grant from the U.S. Department of the Interior (Grant Number G11AP2007).

\section{References}

[1] ITRC, Use and Measurement of Mass Flux and Mass Discharge. $M A S S F L U X-1$. Interstate Technology \& Regulatory Council: Washington, D.C., 2010.

[2] Einarson, M.D., Mackay, D.M., Predicting impacts of groundwater contamination. Environmental Science and Technology, 35(3), pp. 66A73A, 2001.

[3] Bockelmann, A., Zamfirescu, D., Ptak, T., Grathwohl, P. and Teutsch, G., Quantification of mass fluxes and natural attenuation rates at an industrial site with a limited monitoring network: a case study. Journal of Contaminant Hydrology, 60(1-2), pp. 97-121, 2003.

[4] Hatfield, K., Annable, M., Cho, J., Rao, P.S.C. and Klammler, H., A direct passive method for measuring water and contaminant fluxes in porous media. Journal of Contaminant Hydrology, 75(3-4), pp. 155-181, 2004.

[5] Annable, M., Hatfield, K., Cho, J., Klammler, H., Parker, B., Cherry, J. and Rao, P.S.C., Field-scale evaluation of the passive flux meter for simultaneous measurement of groundwater and contaminant fluxes. Environmental Science and Technology, 39(18), pp. 7194-7201, 2005.

[6] Klammler, H., Hatfield, K., Annable, M., Agyei, E., Parker, B., Cherry, J. and Rao, P.S.C., General analytical treatment of the flow field relevant to the interpretation of passive flux meter measurements. Water Resources Research, 43(4), W04407, 2007.

[7] Strack, O.D.L., Groundwater Mechanics, Prentice Hall: Englewood Cliffs, N.J., 1989. 